\title{
High performance diamond-like carbon layers obtained by pulsed laser deposition for conductive electrode applications
}

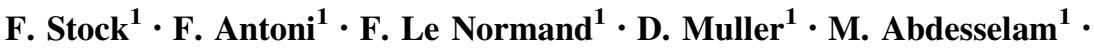 \\ N. Boubiche ${ }^{2}$ I. Komissarov ${ }^{3}$
}

Received: 21 June 2017/Accepted: 16 August 2017/Published online: 21 August 2017

(C) Springer-Verlag GmbH Germany 2017

\begin{abstract}
For the future, one of the biggest challenge faced to the technologies of flat panel display and various optoelectronic and photovoltaic devices is to find an alternative to the use of transparent conducting oxides like ITO. In this new approach, the objective is to grow high conductive thin-layer graphene (TLG) on the top of diamond-like carbon (DLC) layers presenting high performance. DLC prepared by pulsed laser deposition (PLD) have attracted special interest due to a unique combination of their properties, close to those of monocrystalline diamond, like its transparency, hardness and chemical inertia, very low roughness, hydrogen-free and thus high thermal stability up to $1000 \mathrm{~K}$. In our future work, we plane to explore the synthesis of conductive TLG on top of insulating DLC thin films. The feasibility and obtained performances of the multi-layered structure will be explored in great details in the short future to develop an alternative to ITO with comparable performance (conductivity of transparency). To select the best DLC candidate for this purpose, we focus this work on the physicochemical properties of the DLC thin films deposited by PLD from a pure graphite target at two wavelengths (193 and $248 \mathrm{~nm}$ ) at various laser fluences. A surface graphenization process, as well as the required efficiency of the complete structure (TLG/DLC)
\end{abstract}

\section{F. Stock}

francois.stock@etu.unistra.fr

1 ICube, D-ESSP, 23 rue du Loess, 67037 Strasbourg, France

2 Laboratoire d'etude physico-chimiques des matériaux (LEPCM), Dép. de physique, Faculté des sciences de la matière, Université Batna1, 05 Avenue Chahid Boukhlouf, 05000 Batna, Algeria

3 Belarusian State University of Informatics and Radioelectronics, P. Brovka str. 6, 220013 Minsk, Belarus will clearly be related to the DLC properties, especially to the initial $\mathrm{sp}^{3} / \mathrm{sp}^{2}$ hybridization ratio. Thus, an exhaustive description of the physicochemical properties of the DLC layers is a fundamental step in the research of comparable performance to ITO.

\section{Introduction}

Over the last decades, different forms of carbon including inorganic carbon compounds emerged as a new class of materials possessing remarkable properties and, accordingly, a wide range of potential technological applications. PLD proved to be one of the most successful preparation techniques, being able to fabricate all members of this class $[1,2]$. DLC (also named tetrahedral amorphous carbon: taC) is one of the most interesting material thanks to the very close physicochemical properties to those of diamond. Indeed, transparency and hardness are very similar to diamond but due to the very different production ways, the material can only be produced as thin films and always present an amorphous structure. The adamant character of the layers is directly related to the inner $\mathrm{sp}^{3} / \mathrm{sp}^{2}$ hybridization ratio $[3,4]$. In addition, the surface roughness is also very low and the insulating character is very high. PLD is a very powerful technique to produce DLC with a very high adamant character. The second biggest advantage of this technique is the ability to produce hydrogen-free DLC which is very important when the use of surface laser treatments is planned. The presence of hydrogen in DLC is more commonly related to CVD-produced films [5]. It is also important to notice that the presence of hydrogen does not improve the $\mathrm{sp}^{3}$ hybridization of the carbon atoms. The highest measured $\mathrm{sp}^{3}$ concentration is a specificity of the DLC layers deposited by the PLD technique and can reach 
values close to $90 \%$ as it is already shown in the literature [6]. It is also well known that the adamant character of DLC obtained by PLD is also related to the laser wavelength. The most efficient sources are excimer-pulsed lasers. In this work, we will make a direct comparison of the DLC layers properties deposited in the same conditions at two laser wavelengths: 193 and $248 \mathrm{~nm}$.

\section{Experimental}

Compared to many other thin film deposition techniques, PLD is relatively easy to implement. The experimental setup we use for this experiment is quite conventional. A focused laser beam is introduced in a vacuum chamber through a quartz window and hits a graphite target $(99.99 \%$ purity) with a $45^{\circ}$ angle of incidence. The matter is ablated perpendicularly to the target, which is rotated at $1 \mathrm{rpm}$ to avoid the formation of deep craters at its surface. Consequently, successive laser pulses are always focused on a clean polished graphite surface. The evaporated matter produces a plasma plume in which the atomic species, mostly composed of ionized carbon atoms, acquires kinetic energy up to a few hundred of $\mathrm{eV}$. The collected species are condensed on a substrate placed parallel to the target surface at a fixed distance of $5 \mathrm{~cm}$. It is important to notice that the substrate is fixed in optimal condition to obtain the most uniform layer thickness. For all the described experiments, all substrates are placed at the same position. The laser source (LAMBDA PHYSIK Compex 201) produces two different wavelengths: 193 or $248 \mathrm{~nm}$ while using $\mathrm{ArF}$ or $\mathrm{KrF}$ excimer gas mixtures, respectively. At $193 \mathrm{~nm}$, the available energy is slightly reduced due to the laser light absorption in air and the related production of ozone. This undesirable effect limits the use of very high fluences when compared to $248 \mathrm{~nm}$ emission. The pulse duration is also slightly different, changing from $20 \mathrm{~ns}$ for ArF to $25 \mathrm{~ns}$ when using $\mathrm{KrF}$. It is also important to notice that the fluence were very precisely measured for each wavelength by searching the most focusing lenses position upstream the vacuum chamber. The laser spot on the graphite target is, therefore, always optimal. To ensure very precise microscopic impact measurements, silicon and aluminium polished surfaces were substituted to graphite at the target position and guarantee that way a reliable value of the calculated fluence. The PLD chamber is equipped with a turbomolecular pump which allows to reach a remaining background pressure in the $10^{-8}$ mbar range. As it was already shown $[7,8]$, the highest adamant character is obtained at room temperature. Therefore, no heating of the substrates is necessary during the deposition process. DLC thin films are deposited on quartz substrates when optical measurements of transmission are necessary.
However, most of the physicochemical properties presented in this work do not need a transparent substrate.

\section{Results and discussion}

\subsection{Film thickness}

One of the specificity of the PLD is that the deposition rates are low enough to be easily controlled by the number of pulses. Thus, it is easily possible to deposit one or a few equivalent carbon monolayers with a high degree of precision. In our work, especially because we plan to graphitize a few numbers on atomic layers on top of the DLC surface, we have deposited in preliminary experiments on quartz or $\mathrm{MgO}$ substrates, an amount of carbon equivalent to 1,3 and 6 graphite monolayers (GML). To graphitize this matter, we performed a thermal annealing at $700{ }^{\circ} \mathrm{C}$ for $2 \mathrm{~h}$. Even if the microscopic layers do not show a good uniformity, it has to be noticed that experimental and theoretical values of transmittance are very comparable, confirming the ability of the technique to control the amount of the deposited matter with a very good precision. These results are presented in Fig. 1. To highlight formation of GML, Raman spectroscopy was performed on the treated samples. Results are presented in Fig. 2. We clearly identify the presence of graphene regarding to the $2 \mathrm{D}$ band. In this way, Raman spectra complete transmittance measurements showing the presence of graphene especially for 1 GML.

We were mainly depositing the DLC layers on oxidized silicon $<100>\left(63 \mathrm{~nm}\right.$ of $\left.\mathrm{SiO}_{2}\right)$. This choice is not only due to an economical reason but also because the substrate surface remains very close to the original Si wafer roughness guaranteeing thereby very precise thickness measurements as obtained by profilometry (DEKTAK 150 and Talystep). The laser fluence is adjusted between 2 and

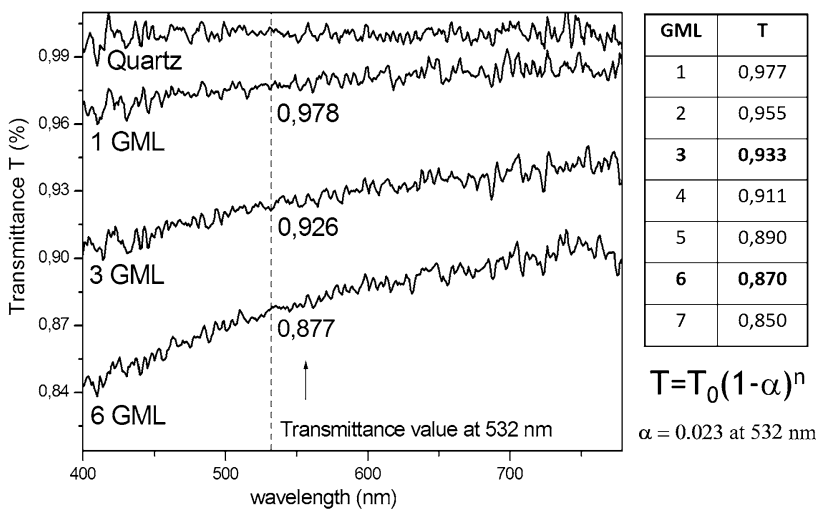

Fig. 1 Transmittance measurements as a function of the deposition duration $(40,120$ and $240 \mathrm{~s}$ at $10 \mathrm{~Hz}$ repetition rate) with a $193 \mathrm{~nm}$ laser (fluence $=4.7 \mathrm{~J} / \mathrm{cm}^{2}$ ) and compared to theoretical values 
$10 \mathrm{~J} \mathrm{~cm}^{-2}$ but remains limited to $7 \mathrm{~J} \mathrm{~cm}^{-2}$ in the case of ArF for the reason explained earlier. An important effort was performed to prepare layers presenting precisely controlled thickness. For each fluence used in the setup, a specific calibration was made by extracting the specific deposition rate to grow films with a very constant thickness of $20 \mathrm{~nm}$. This choice was made to obtain with a very good precision, the density of our layers as deduced from nuclear reaction analysis (NRA) measurements. Figure 3 shows that the deposition rates at $248 \mathrm{~nm}$ are almost a factor 2 higher compared to $193 \mathrm{~nm}$ and this, for all fluences. In addition, the evolution is almost linear for both wavelengths. However, we must keep in mind that the kinetic energy of the ablated species present in the plasma are much higher at the shortest wavelength as also

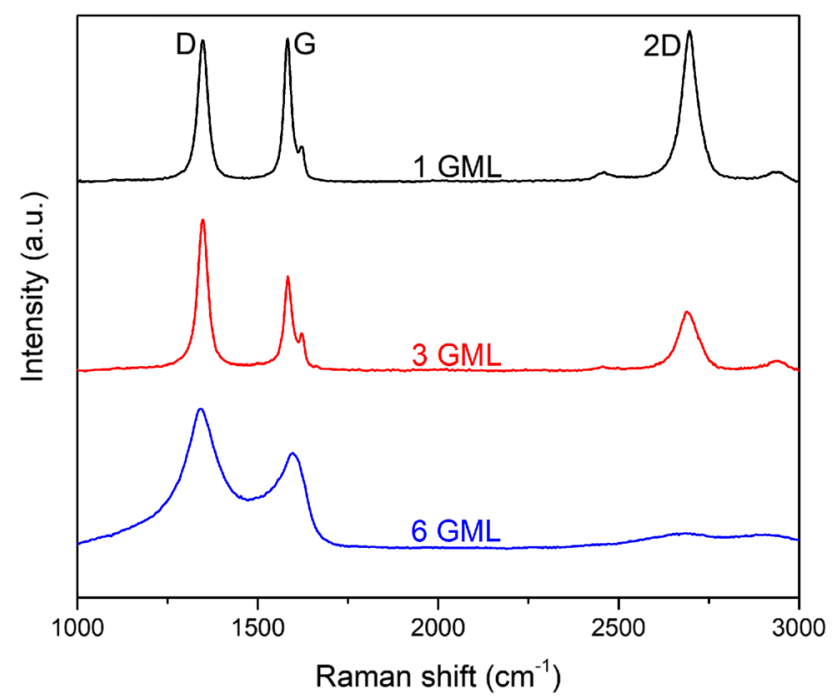

Fig. 2 Normalized Raman spectra obtained for 1, 3 and 6 GML

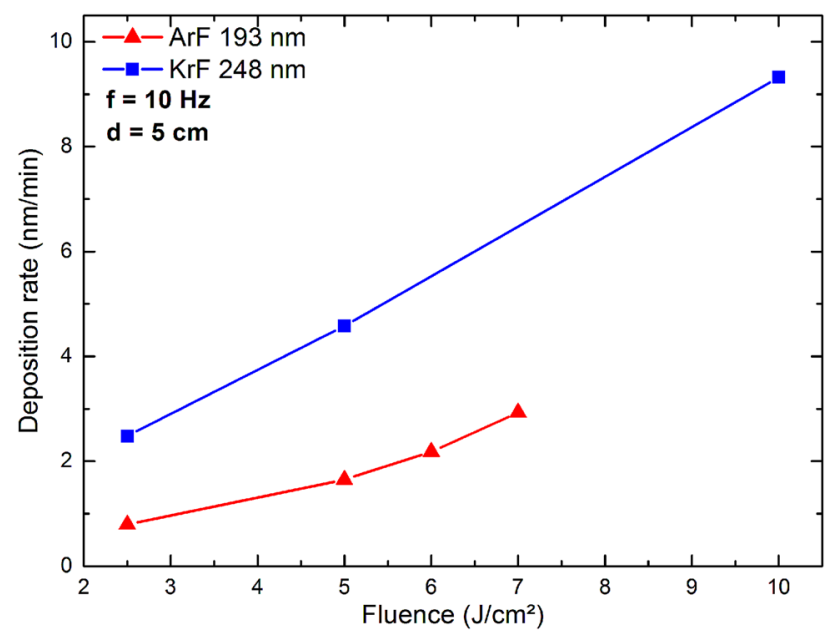

Fig. 3 Deposition rate evolution for DLC thin films as a function of fluence for 193 and $248 \mathrm{~nm}$ PLD of graphite demonstrated in previous works [9-11]. All deposited DLC thin films were also analysed by Raman spectroscopy. Because almost all measured spectra do not show any significant differences, we will not present the spectra here. The interpretation of the Raman analysis is not yet easy to correlate to the presented thin film properties.

\section{$3.2 \mathrm{Sp}^{3} / \mathrm{sp}^{2}$ concentration}

To obtain the $\mathrm{sp}^{3}$ relative concentration in the deposited DLC layers, the films were characterized by X-ray photoemission spectroscopy (XPS) and electron-energy loss spectroscopy (EELS). The XPS spectra, not shown here, do not present any trace of oxygen or undesirable contaminant in our layers. The $\mathrm{sp}^{3}$ concentration as a function of the fluence at 193 and $248 \mathrm{~nm}$ is presented in Fig. 4. It can be observed that the values measured at $193 \mathrm{~nm}$ are much higher compared to the concentration in the layers deposited at $248 \mathrm{~nm}$. It is also important to notice that it clearly seems that an optimal value of the fluence appears around $5 \mathrm{~J} \mathrm{~cm}^{-2}$. The same behaviour is observed for both wavelengths even if the maximal value of the $\mathrm{sp}^{3}$ concentration is reaching $85 \%$ at $193 \mathrm{~nm}$ but does not exceed $62 \%$ while working with the $\mathrm{KrF}$ source. At very low fluence, the atomic carbon present in the plasma does not have enough kinetic energy to enforce the $\mathrm{sp}^{3}$ binding character in the deposited layers. At very high fluence, the atoms reaching the surface present an average energy which is scattered in the deposited layers and force the disintegration of the highly $\mathrm{sp}^{3}$ concentrated film. Therefore, the $\mathrm{sp}^{2}$ hybridization becomes most prominent and enhances the graphitic character of the deposited layer. This effect was already demonstrated by a subplantation model during the film growth [12]. We have to consider

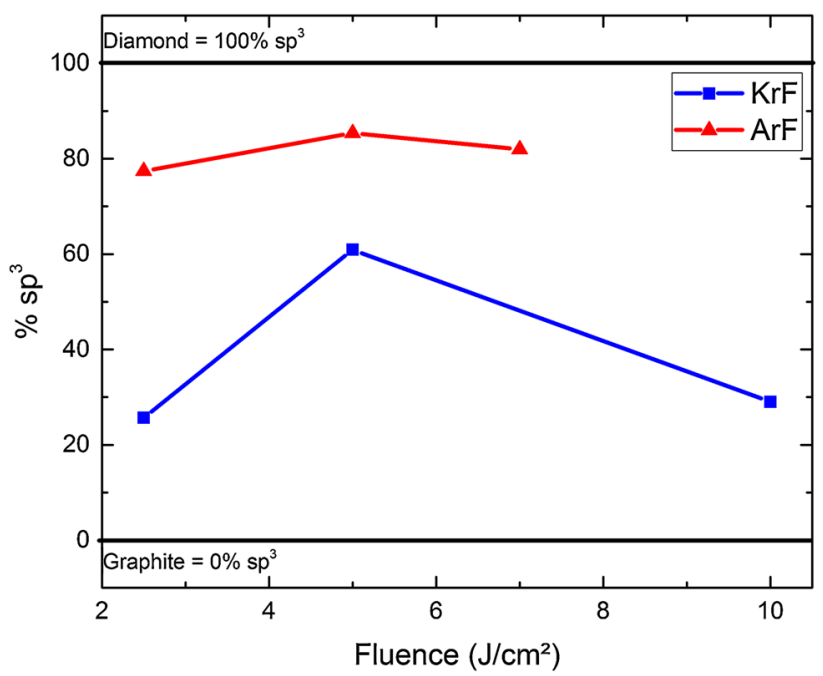

Fig. $4 \mathrm{Sp} 3$ concentration in the DLC thin films as a function of fluence for 193 and $248 \mathrm{~nm}$ PLD of graphite 
that the XPS measurements are significant for the first atomic layers on the surface only. Therefore, the strong graphitization effect at very high fluences cannot be representative of what happens in the whole DLC layers. This must be considered when the graphitization level is compared to thermally treated thin films in which the temperature is homogeneous during the annealing process.

\subsection{Density measurements}

NRA measurements were performed using a $900 \mathrm{keV}$ deuterons beam. The density of our layers was deduced knowing the precise thickness of our layers. As it is shown on Fig. 5, a direct correlation between the evolution of the density and the $\mathrm{sp}^{3}$ concentration in the DLC layers is observed for both wavelengths. In the case of the $\mathrm{KrF}$ deposited layers, the density is varying between 1.8 and $2.7 \mathrm{~g} \mathrm{~cm}^{-3}$ depending on the fluence. The same behaviour is also observed for thin films deposited at $193 \mathrm{~nm}$ with a very noticeable value at the optimal fluence of $5 \mathrm{~J} \mathrm{~cm}^{-2}$. At this specific fluence, the density reaches $3.46 \mathrm{~g} \mathrm{~cm}^{-3}$ which is very near to the value of crystalline diamond $\left(3.57 \mathrm{~g} \mathrm{~cm}^{-3}\right)$. The adamant character of the amorphous DLC films is, therefore, confirmed for the optimal fluence of $5 \mathrm{~J} \mathrm{~cm}^{-2}$ and is much more prominent at the wavelength of $193 \mathrm{~nm}$. At higher fluences, the density decreases drastically, in good agreement with the $\mathrm{sp}^{3}$ concentration. It can be noticed that the variation follows almost the same trend in both cases. For the $\mathrm{KrF}$ case, for example, when the $\mathrm{sp}^{3}$ fraction is reduced by $25 \%$, the corresponding density is also decreasing by the same order if we consider $100 \%$ density is pure diamond.

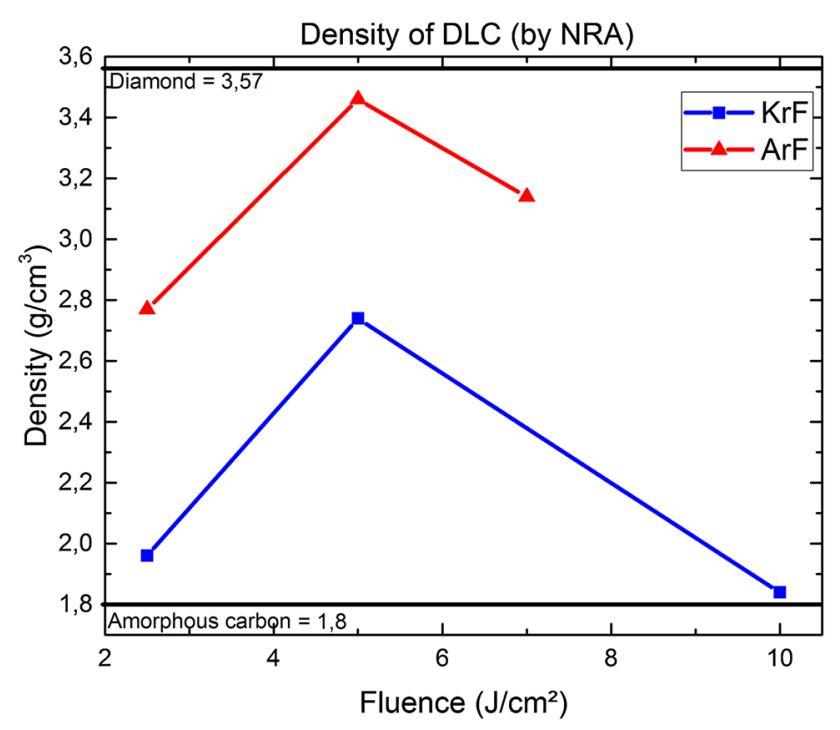

Fig. 5 DLC thin films density obtained by NRA as a function of fluence for 193 and $248 \mathrm{~nm}$ PLD of graphite

\subsection{Optical properties}

Refractive index $n$ and extinction coefficient $k$ are deduced from spectroscopic ellipsometry measurements (HORIBA JOBIN-YVON). The different results reported in Fig. 6, corresponding to different experimental conditions, are based on the Tauc-Lorentz model [13]. It is not always easy to compare to other published results even if our results are in very good accordance with many other previous works $[14,15]$. However, a few important observations can be deduced from our results. First, the $\mathrm{ArF}$ deposited layers present in any case a higher $\mathrm{n}$ index and a lower $\mathrm{k}$ coefficient when compared to KrF deposited DLC. This result can also be interpreted as the higher adamant character of the ArF deposited layers. All films show the same trend except for the unique case of the film deposited
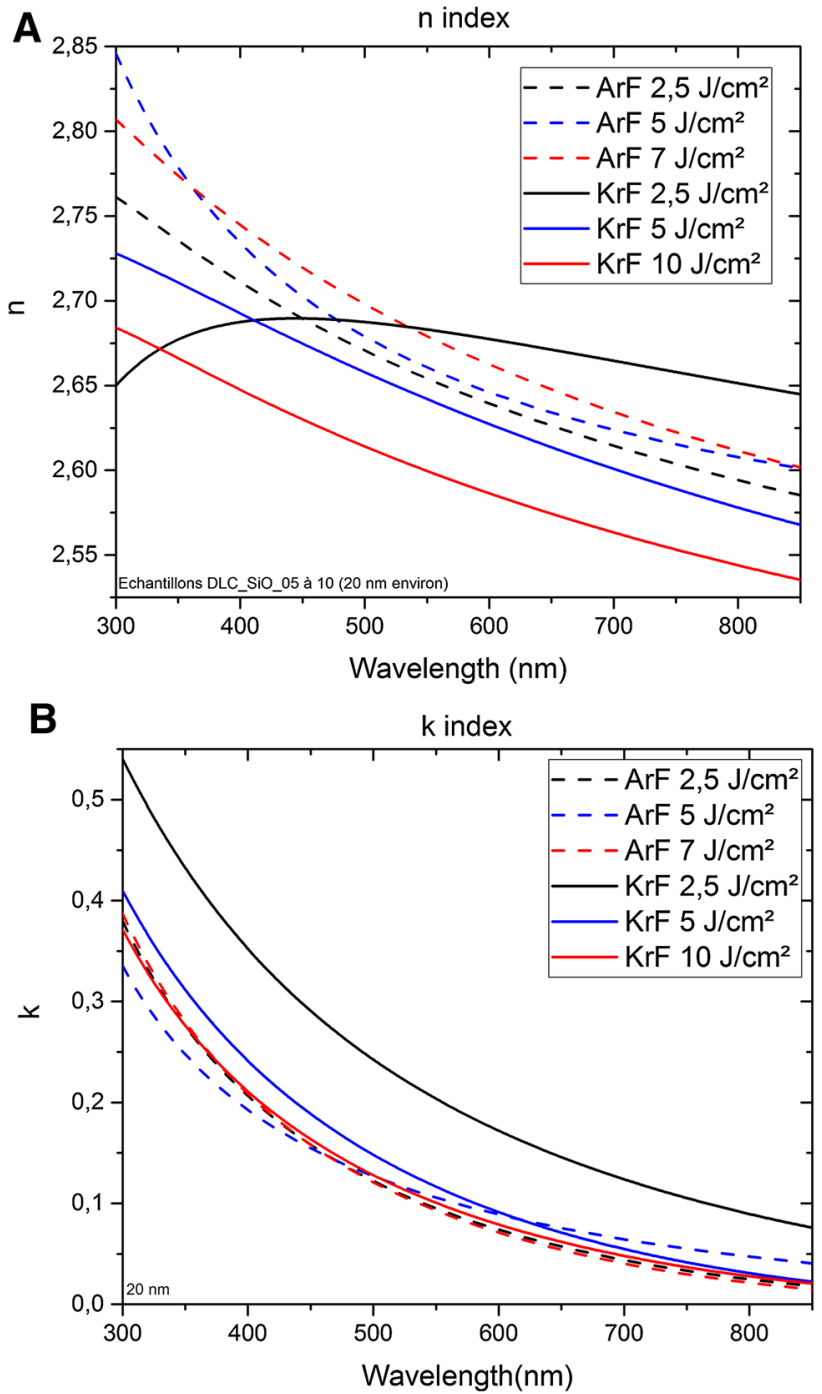

Fig. 6 Refractive index $\mathrm{n}$ and extinction coefficient $\mathrm{k}$ as a function of wavelength for DLC thin films deposited by PLD (193 and $248 \mathrm{~nm}$ ) as a function of fluence 
at $248 \mathrm{~nm}$ at the lowest fluence. We could think on an artefact of the model used for the index calculation. However, it can also be noticed that similar results were already published in the case of DLC layers deposited by other techniques in which the kinetic energy of the atomic species leading to a film growth are lower compared to a few hundred of $\mathrm{eV}$ as it is the case in PLD using excimer lasers. We can also notice that the extinction coefficient is close to 0 in the visible range, confirming that all the layers are transparent in the visible range. More important is to notice that $\mathrm{k}$ is increasing drastically when reaching wavelength values under $300 \mathrm{~nm}$, making the layer opaque to UV light. This can clearly orientate us for the choice of the laser sources which have to be used for future surface laser annealing of the DLC.

\section{Conclusion}

In this work, we have directly compared DLC thin film properties obtained by PLD for two laser wavelengths, 193 and $248 \mathrm{~nm}$, respectively. The different thin films were produced at various fluences but in the same experimental conditions, except laser wavelength which was modified to compare the physicochemical properties of the produced DLC layers. The aim of our work is to select the best candidate to be used as a transparent and insulating substrate for the growth of TLG on top of it. The final multilayered structure will produce a transparent and conductive which can be used as an alternative to TCO like with comparable performance to ITO.

There is a clear effect of the laser wavelength to the $\mathrm{sp}^{3}$ concentration in the layers. At any fluence, the ArF gas mixture, producing a laser beam at $193 \mathrm{~nm}$, leads to the growth of thin films with a very high adamant character. In the best case, the $\mathrm{sp}^{3}$ concentration is close to $85 \%$ and the density of the film is reaching $97 \%$ of that of diamond as it is deduced by XPS and NRA measurements. It is also observed that the best transparency in the visible range (lowest $\mathrm{k}$ coefficient) is also obtained for these conditions as well as the very high value of the refraction $n$ index, confirming also the adamant character of the films. DLC being a metastable state of carbon, we believe that the best candidate to be used as transparent substrates to produce pure carbon electrodes must be the layers originally produced with the highest concentration of $\mathrm{sp}^{3}$ hybridization. We have already shown in previous works that those layers have also the best stability when thermal or laser posttreatments are made to obtain a partial graphitization of the films [7, 8]. Finally, we can conclude that whatever the experimental conditions are, $\mathrm{KrF}$-produced thin film are not able to reach the adamant character level of the ArFproduced DLC layers.

\section{References}

1. J. Perriere, E. Millon, E. Fogarassy (eds.), Recent Advances in Laser Processing of Materials, 1st edn, E-MRS Series (Elsevier, New York, 2006)

2. D.B. Chrisey, G.K. Hubler (eds.), Pulsed Laser Deposition of Thin Films (Wiley, New York, 1994)

3. J. Robertson, Mater. Sci. Eng. R37, 129 (2002)

4. A.C. Ferrari, Surf. Coat. Technol. 180-181, 190 (2004)

5. J. Robertson, Tribol. Int. 36, 405 (2003)

6. Y. Lifshitz, Diam. Relat. Mater. 8, 1659 (1999)

7. S. Rey, F. Antoni, B. Prévot, E. Fogarassy, J. Arnault, J. Hommet, F. Le Normand, P. Boher, Appl. Phys. A 71, 433 (2000)

8. Z. Nibennaoune, D. George, F. Antoni, S. Ahzi, D. Ruch, J. Gracio, Y. Rémond, Diam. Relat. Mater. 22, 105 (2012)

9. D.T. Peeler, P.T. Murray, AIP Conference Proceedings 288, Laser Ablation: Mechanism and Applications II. 248, 359 (1993)

10. D.H. Lowndes, V.I. Merkulov, A.A. Puretzky, D.B. Geohegan, G.E. Jellison, C.M. Rouleau, T. Thundat, MRS Proceedings (MRS, Warrendale, 1998), p. 325

11. A.A. Puretzky, D.B. Geohegan, G.E. Jellison Jr., M.M. McGibbon, Appl. Surf. Sci. 96-98, 859 (1996)

12. J. Robertson, Diam. Relat. Mater. 3, 361 (1994)

13. G.E. Jellison, F.A. Modine, Appl. Phys. Lett. 69(3), 371 (1996)

14. G. Reisse, B. Keiper, S. Weissmantel, U. Falke, Appl. Surf. Sci. 127-129, 500 (1998)

15. K. Honglertkongsakul, P.W. May, B. Paosawatyanyong, Diam. Relat. Mater. 19, 999 (2010) 